\title{
Research on Education Process Fairness from the Perspective of Differential Education
}

\author{
Xiang Li \\ School of Education \\ Nanjing Normal University \\ Nanjing, China 210023 \\ Language College \\ Nanjing Normal University of Special Education \\ Nanjing, China 210038
}

\begin{abstract}
Education process fairness aimed at students' individual needs adopts a reasonable differential treatment. It is the reflection and application of fair principle and fair value in the process of education. Education process fairness is in the pursuit of all-round development of students, and adopts different methods in the process of education according to students' individual differences to satisfy the rational needs of the students' development. Theory of Multiple Intelligence reveals that each student has different degrees of multiple intelligences. Education should meet the development needs of students' multi-intellectual abilities. Namely differential education should be implemented in the process of education to develop the students' potential to the maximum. This is just a fair education process.
\end{abstract}

Keywords-education process fairness; multiple intelligences; differential education

\section{INTRODUCTION}

Education Fairness is a hot topic of contemporary society. Education fairness is the foundation of social justice, playing a promoting role in the social stratified flow. People from the ordinary students to academic researchers are very concerned about education fairness, and the researchers have carried out the analysis from different perspectives. But the vast majority of scholars focus on the macro point of view to think about education fairness, and label it as social fairness, which is conditioned by social justice and social development to a certain extent. However, the real insight into the meaning of the education fairness is only to put it to the micro level to make a breakthrough. The education fairness can dynamically be divided into starting fairness, process fairness and result fairness. The fundamental purpose of education fairness is all-round development of students' individual, which adopts reasonable differential treatment based on the students' individual characteristics. Education process fairness reflects "the optimization development of different students' individual" which is the essential

This research sponsored by Jiangsu Provincial Department of Education Qing Lan Project.

Xiang Li, Ph.D candidate of School of Education, Nanjing Normal University \& Associate professor of Language College, Nanjing Normal University of Special Education. connotation of education fairness in the process of education.

\section{The BASIC CONTENTS OF EDUCATION PROCESS FAIRNESS}

According to Whitehead's Process Philosophy, process is a way of existence of things, and the existence of process constitutes the essence of the world. Without the process, things can't exist. Engels has said: "The world is the aggregate of process ". German educator Wolfgang Brezinka has put forward: "education is a 'process concept' rather than a kind of 'product concept'. Education is a process or experience of a guiding." As an educational activity, the form of education is a process, so process should be the basic attribute.

Education process fairness is different from education fairness. Education fairness has the macroscopic and microscopic two aspects. Macro level of the education fairness refers to the whole education system fairness such as the education resource allocation fairness, which is from the sociological perspective; Micro level of the education fairness mainly refers to the internal fairness such as the education process fairness, which is from the educational perspective. Macro level of fairness is built on the meaning of "content", lacking a fair understanding and reflection of "man". Only concerning micro-level fairness, namely education process fairness, can various external affecting factors contributing to education fairness take effect.

In the education practice, education process fairness should be as follows:

Firstly, educators should be fair to the students in the process of education, and promote the optimization development of different students' individual through various means. Provide suitable education according to students' age, ability, qualifications and so on. Education process fairness focuses on how to make students obtain "deserved things", and furthermore this way of obtaining is justified. Although each student hopes to obtain "deserved things" in a fair way, "deserved things" is uncertain inevitably. A person's "deserved things" mostly depend on the distribution and allocation. No matter what kind of equity theory is, therefore, 
the connotation of "deserved" and the explanation of allocation situation should be given firstly. We can't make abstract judgment out of the objective environment of the existence of things. In terms of education process fairness, "deserved things" refers to the education value, namely the social value provided by the institutionalized education. It specifically refers to what satisfy the wants of students' individual all-round development. The standard of "deserved" is to meet the reasonable needs of the students' development. Thus, the education process fairness is a kind of reasonable equality. In Ethics and Education British education philosopher Peters has pointed out that "equality" is a term used to compare and has two results: equality or inequality. "Everyone is equal" refers to the equal treatment to all, rather than the way of treatment to all is the same. The conclusion is in accordance with the ancient Greek philosopher Aristotle's view: Treat the equal fairly, the unequal unfairly. Thus it says clearly how to treat the two properties of fairness: The same things should be treated equally; Different things should be treated differently.

Secondly, "to ensure that every person can get the appropriate education and this kind of education method and schedule is suitable to individual characteristics". Fairness doesn't mean treating a diversity of individuals in the same way. The real fairness is consistent with the principle of teaching students according to their aptitude. It is not equalitarianism. It is not unfair to implement different standards of syllabus for good and poor students. Instead, it is unfair that the class teaching process just meets needs of medium level students, except the excellent students and the backward students. Class teaching system focuses on teaching synchronization, standardization and unification, and the same treatment to differences. The rigid identity constrains the development of students' personality. Therefore, the education process, ignoring the students' individual differences and personality developments, showing no difference in different students, is a fairness on the surface, essentially unfair. Fairness should include two levels: the equal treatment and the differential treatment. In the modern "people-oriented" education situation, the core of education process fairness should be how to treat students differently.

In general, the education process fairness must not be understood in one-sided way: cut the peak or eliminate difference. Otherwise it will influence and even endanger the individual development along with all-round development of all students. As educator John Dewey points out: All the people imposed on one established method of the unity will be the universal mediocrity, very few exceptions. Education process fairness shows care for students' different learning needs, reflecting a kind of humanistic thought. The fundamental purpose of education process fairness is to offer the education opportunities and conditions suitable to the development of each student, helping students' individual get full development. It is based on the respect for each student on the basis of individual differences, paying attention to the demand of different individuals.

\section{DifFERENTIAL EDUCATION - THE MiCRO LEVEL IMPLEMENTATION OF EDUCATION PROCESS FAIR}

\section{A. The Core Connotation of Differentia Education}

In the 1980s, the United States Harvard University psychology professor Howard Gardner put forward the theory of Multiple Intelligences, in which "intelligence" refers to the ability to solve problems in real life, as well as the ability to provide valuable services and creation in one's culture. Intelligence includes eight kinds intelligence: language intelligence, music intelligence, logical mathematical intelligence, spatial intelligence, body kinesthetic intelligence, personality, intelligence, selfcognitive intelligence and natural cognitive intelligence. Gardner's research shows that combination is the form of intelligent operation, and any experienced adults will use the combination of intelligence to solve practical problems. For students, multiple intelligences have their own characteristics, and shows in a unique form. Social individuals provide valuable services and make contributions to human culture in their own unique way. The primary task of education is to discover the students ' weak intelligence and develop their strengths. The Theory of Multiple Intelligences advocates the difference of human intelligences mainly lies in the different intelligence combinations among different people. A person may not appear talented in any intelligence, but may play a certain role well if all the skills and techniques are cleverly combined. Therefore, any strong intelligence needs to be cleverly combined with other intelligences. Guided by this theory, differential education acknowledges the particularity and differences of students' intelligences, along with the nonsynchronicity of their cognitive abilities in different fields. It encourages us to educate according to students' different intelligences, create suitable education methods based on students' acceptable ability, and thus promotes the development of students' different multiple intelligences.

The core of the differential education can be summarized as: "Seek differential development" — the goal, the kernel and the foothold of differential education, "Start from the differences" - the starting point and basis of differentia education, "Implement differential teaching" — the means and ways of differentia education. That is to say, The goal of differential education should be to promote the students' "deserved" differential and individual development on the basis of common development; Education activities should respect the individual differences of students, maintain or develop a reasonable and positive difference, reduce or eliminate the unreasonable and the negative differences among the students' individual, with the differences of students' individual as the premise and starting point; In the process of education, different teaching contents, different teaching methods, different evaluation should be made, and different education activities should be organized in terms of the differences of students, in order to realize the diversity development of students. 


\section{B. Differentia Education -the Inevitable Choice of Education Process Fairness}

First of all, with the popularization of compulsory education, the differences of students' individuality increase. Especially the application of "an exemption to the nearest school" policy reflects the education starting fairness in the compulsory education stage, but students have great individual differences in the same class because their learning foundation and the characteristics can't be considered when their schools or classes are divided as a result of the lack of unified exam. In addition, in the compulsory education stage, students can't resume course in spite of their unqualified academic performance, so the gap among students often gradually increases with the rising of grade. In the process of education, to promote full development of each student on the basis of his own original level, educators should implement differential education to satisfy the needs of all students.

Secondly, in order to reflect the equality of all the basic human rights of students, education process fairness should ensure all the students participate in the education activities, avoiding the rejection of the students with special needs. What's more, inclusive education, demanding a combination of special education and general education, requires the unification of the education system construction as an organic whole, so as to meet the learning needs of all the students. In this situation, it's common that the students with special needs enter the regular school classes for further study. Thus the differences among class students' individual are undoubtedly magnified. In the education process, educators can only really implement differential education to treat special students and normal students fairly.

Thirdly, in modern society, with the quickening industrialization and urbanization, a large number of migrant workers have emerged into the cities, and then their children have also entered the local schools to accept education. Due to the weak cultural foundation, the language communication barrier and the changed cultural environment, differences between this group and class students have appeared inevitably. Therefore it's bound to carry out differential education to help them integrate into the class and make all the students get full development.

It's often seen in the vulnerable groups that their families' social status is more or less low, their families' members' cultural qualities are not satisfying, and even their parents don't attach importance to education. Besides, there are other factors unfavorable to students' academic development, which may lead to the transfer of intergenerational. Therefore, to get students with special needs and students with financial difficulties back to school, the simple adoption of the economic measures to compensate will not ensure that they get fully developed. Only through the implementation of differential education in the education process, to reduce or eliminate the adverse factors contributing to the development of students and make the students from different environment as well as family background get the differential development to compensate the disadvantaged groups, can the deep connotation of differential education be reflected. Thus it is a higher level of education process fairness.

\section{THE IMPLEMENTATION OF EDUCATION PROCESS FAIRNESS-BASED ON DIFFERENTIAL EDUCATION}

Fairness is concealed in the whole education process, and students' emotional experience concerning education process fairness is caused by many invisible factors such as teachers' teaching methods, classroom organization, and teachers' personalities and so on. It is largely determined by the educators' personal ideas and behavior, but the implementation of differentia education mostly requires educators' fair behavior:

\section{A. Focus on Differences; Teach on the Basis of Students}

The theory and practice of education indicates that the students' knowledge, abilities, personalities and so on have various differences. The former Soviet Union educator Babanski thinks a class of one hundred students should have one hundred kinds of learning motivation. Gardner's Theory of Multiple Intelligences has also proved that the differences among individual intelligence do exist, and the form of the differences has diversification. Each student individual has its unique value, and the differences of students' individual should be recognized. Education is supposed to offer the students' individual equal opportunities in the pursuit of education diversity based on the characteristics of each student.

The differences among students' individual mainly are reflected in intelligent structure, self-consciousness and initiative. Research shows that individual's mental is the outspread of his society's psychological activity, associated with certain social cultural background. Meeting a diversity of difficulties during study, students are often at a disadvantage, so they should be treated differently according to different situation and different personalities in the process of education. On the one hand, the initiative of the students with strong subjectivity should be exerted; On the other hand, targeted measures should be taken to mobilize the positive initiative of the students with weak subjectivity. In the education practice, the American scholar Coleman's Compensation Principle of Equality and Rae's Discriminatory Principle can be taken into consideration: corresponding teaching and education modes are taken based on the students' learning basis, learning habits, learning style, learning interest and so on. Students should be fully understood, and the teaching activities should be designed based on the grasp of students' learning. The differences of students' individual should be focused on during the whole process of education, and differentia treatment should be carried out as well, to promote students' full development.

\section{B. Create Harmonious Interaction; Improve all Kinds of Students Participation}

On the one hand, the harmonious interaction between teachers and students is the psychological foundation of realization of education process fairness, which can arouse the subjective consciousness of students' individual, promote 
the development of the students' individual, make each student in a group feel respected, have a sense of identity and belonging, as well as keep a good mental state of realizing oneself. Thus, positive emotional attitude towards academic challenges can be generated, which is the inner demand of education process fairness.

On the other hand, since no one of the students is out of classroom learning, effective classroom participation is an important guarantee for students' individual development. Classroom participation includes cognitive engagement, emotion engagement and behavior engagement, and each kind of participation is an important impact on the development of students. All the students' participation attitude, participation opportunities and participation effect should be concerned. In addition, the participation opportunities for each students should be created, and a variety of free choice should be offered according to the students' individual differences in the classroom, such as the choice of learning content, the choice of learning mode, the choice of the ways of evaluation, etc., so that each student can get education suitable to his needs of growth and development.

Interaction between teachers and students as well as the teaching participation of students reflect that students' status gets respected and students' right gets recognized. It is an important characteristic of education process fairness.

\section{Teach Differently; Evaluate Multivariately}

Differential teaching embodies the principle of individualized teaching, that is, arrange teaching activities according to the actual demand of students in the process of education, in accordance with the students' existing level and ability, in order to make them get all-round development. Differential teaching is established in all students, advocates to determine different teaching goals and appropriate teaching activities in terms of the students of different levels. The "appropriate" here refers to conforming to the students' current level of intellectual development, following their cognitive characteristics, personality characteristics and individual development, and promoting the development of their potential ability. Differential teaching means making full use of otherness and cooperativeness of different types of students, forming interaction and excitation between educators and students, and promoting the full development of different students. Differential teaching based on the differences of students' individual is an effective way to promote students' differential development.

The teaching process can be controlled through multiple evaluations, namely diversification of evaluation standard, diversified evaluation subject, diversified evaluation content and variety of evaluation methods. Meanwhile students can also be stimulated to participate in teaching actively by multiple evaluations, which aim to encourage students to understand themselves, tap potential, improve learning and build confidence. Multiple evaluations based on students' individual differences focuses on students' differential development, plays the guidance function, incentive function and education function, and in the end develops the potential of each student.

\section{CONCLUSION}

Education process fairness is somewhat like "Utopia", which we can gradually approach, but can't reach. To achieve or be close to education process fairness, thinking about a lot of problems will never end. This is because that the process of education itself contains many conditions and some uncontrolled factors, which determines the breadth, depth and difficulty of education process fairness. Some problems in the education process fairness are the inevitable outcome of the social progress, and some problems are caused by teachers' values and personal qualities, which can be avoided. This is what we should care for when dealing with education process fairness.

\section{REFERENCES}

[1] Whitehead. Process and Reality, Yang Fubin translates, China City Press, 2003, page 13

[2] Deng Yinchen. The Education Fairness and The Social Stratification Flow Function of Education, Education Research and Experiment, 2012(3), page 32

[3] Marx, Engels. Marx And Engels Anthology, People's Publishing House, 1995, page 30.

[4] Wolfgang Brezinka. The Basic Concept of the Education Science Analysis, Criticism and Suggestions, East China Normal University Press, 2001, page63.

[5] The UNESCO International Education Development Committee Learn to Survive - Today And Tomorrow of Education, Foreign Education Laboratory of Shanghai Normal University translates, Shanghai Translation Press, 1979, Page201.

[6] Gardner, H. The Intelligent Structure, Guangming Daily Press, 1990, Page68-67. 